Check for updates

Cite this: RSC Adv., 2017, 7, 28048

Received 13th March 2017

Accepted 4th May 2017

DOI: $10.1039 / c 7 r a 02991 \mathrm{~h}$

rsc.li/rsc-advances

\section{The significance of a new parameter - plasma protein binding - in therapeutic drug monitoring and its application to carbamazepine in epileptic patients}

\author{
Jing-lin Gao, ${ }^{a}$ Xin-yu Wang, ${ }^{a}$ Jing An, ${ }^{b}$ Chao-hui Du, ${ }^{a}$ Meng-jiao Li, ${ }^{a}$ Hai-yan Ma, ${ }^{a}$ \\ Li-na Zhang, ${ }^{a}$ Jing Bian ${ }^{a}$ and Ye Jiang (D) *a
}

\begin{abstract}
Free drug concentration $\left(C_{f}\right)$ is instantaneous and susceptible to sampling time, which can confuse clinicians and cause them to adjust the dosage regimen. In the present work, we indicated a new parameter, the plasma protein binding (PPB) of clinical plasma samples, and discussed its application in TDM (Therapeutic Drug Monitoring). Furthermore, carbamazepine was selected as a model drug to develop a simple pretreatment method for the determination of PPB, in which $C_{f}$ and $C_{t}$ can be simultaneously analyzed in one plasma sample using a single device. Our results demonstrated that this proposed method exhibited some advantages, including perfect recovery (approximately 100\%) and high precision (CV\% < 3.0\%). Furthermore, it achieved successful application in real plasma samples. Individual differences in PPB (from 10.1-68.9\%) were among patients, and in additon, between the patients and healthy people $(p<0.05)$. Moreover, there was a weak correlation between $C_{t}$ and $C_{f}\left(r^{2}=0.470\right.$ and $p<$ 0.05), so the $C_{f}$ of $C B Z$ cannot be estimated from $C_{t}$. However, the primary cause of the variability of $C_{f}$ is the change in PPB. As a consequence, applying PPB to guide individual dose adjustment is more accurate and more scientific than using $C_{f}$ or $C_{t}$.
\end{abstract}

\section{Introduction}

It is well known that only free drugs can pass through membranes to an active site in order to exert pharmacological activity. ${ }^{1}$ In addition, a good correlation between toxicity and free concentration $\left(C_{\mathrm{f}}\right)$ has been found in clinical patients. ${ }^{2}$ Therefore, the monitoring of $C_{\mathrm{f}}$ of a target drug is more valuable for TDM compared to the total concentration $\left(C_{\mathrm{t}}\right)^{2,3}$ However, $C_{\mathrm{f}}$ or $C_{\mathrm{t}}$ determined in vitro just reflects the drug concentration at a specific time, which cannot reflect the overall level of free fraction during drug disposition. They are susceptible to the sampling time. Likewise, typical peak-valley profiles of $C_{\mathrm{f}}$ or $C_{\mathrm{t}}$ are usually observed after drug administration. ${ }^{4,5} C_{\mathrm{f}}$ or $C_{\mathrm{t}}$ obtained at the time to peak concentration $\left(C_{\max }\right)$ or valley concentration $\left(C_{\min }\right)$ could deviate from the true concentration due to an inaccurate sampling time, as well as individual variations. J. J. Marty et al. have demonstrated that the great percentage fluctuation of $C_{\mathrm{f}}$ was in the range of $192-412 \%$ during the $6 \mathrm{~h}$ dosing interval for four patients receiving valproate $\left(400-800 \mathrm{mg}\right.$ ) as usual, compared to $C_{\mathrm{t}}$ (range 153-

aDepartment of Pharmaceutical Analysis, School of Pharmacy, Hebei Medical University, Shijiazhuang, Hebei Province 050017, China. E-mail: jiangye@hebmu. edu.cn; jiangye1@126.com; Tel: +86-0311-86266069

${ }^{b}$ Department of Pharmacy, Hebei General Hospital, Shijiazhuang, Hebei Province 050051, China
$374 \%){ }^{6}$ The variation in $C_{\mathrm{f}}$ or $C_{\mathrm{t}}$ can result in the incorrect estimation of the effective dose available for each patient in clinical practice. Hence, a more accurate parameter contributing to the clinician's optimised drug dosage regimen is a demand for TDM.

In fact, with protein-bound compounds, the primary cause of the variability of $C_{\mathrm{f}}$ in pharmacology is the change in plasma protein binding (PPB). ${ }^{7}$ The binding of a drug to plasma proteins could be in a steady-state equilibrium under stable disease conditions and constant during a relatively long time, which can reflect the whole process of drug disposition. However, the co-administration of other drugs, pathological conditions and gender of patients can markedly change PPB and directly result in a variation in $C_{\mathrm{f}}$, further influencing the efficacy and toxicity of the drug. ${ }^{8,9}$ Recently, Levy R. H. et al. indicated that an increase in $C_{\mathrm{f}}$ in plasma was associated with hypoalbuminemia (hepatic and renal disease, burns and pregnancy) and competitive binding, as antiepileptic drugs are predominantly bound to albumin. ${ }^{10}$ For this reason, the monitoring of PPB in clinical treatments can highlight changes in $C_{\mathrm{f}}$, but also the variability of the individual physiological condition of patients. As PPB is a reliable index in pharmacology and physiology, PPB should be applied for better individualization of a drug dosage regimen in TDM, rather than $C_{\mathrm{f}}$ or $C_{\mathrm{t}}$, to ensure 
the rationality and safety of drug administration in clinical patients.

However, in recent years, drug-protein binding studies for TDM have rarely been reported in clinical practice. Restricting the development of PPB monitoring in clinics is mainly due to some undesired drawbacks of analytical methods used in the laboratory. Traditional methods generally include two parts: the determination of $C_{\mathrm{f}}$ and $C_{\mathrm{t}}$. In other words, two different pretreatment methods and the corresponding devices were required, such as equilibrium dialysis (ED) or centrifugal ultrafiltration (CF-UF) for $C_{\mathrm{f}}$, but also liquid-liquid extraction (LLE) or protein precipitation (PPT) for $C_{t}$ preparation. ED is time-consuming and also has a possible dilution effect on drug levels as a result of a volume shift. ${ }^{1,9}$ This would lead to decreased sensitivity, which would be difficult for the analysis of clinical samples with low concentrations. In contrast, CF-UF is characterised as a simple and rapid separation. However, the volume of the ultrafiltrate $\left(V_{\mathrm{u}}\right)$ was not accurately controlled and the concentration polarization phenomenon could easily occur in the process. Furthermore, the results of analysis showed deviation from the true values. So, controlling the ratio of the ultrafiltrate to the sample solution in CF-UF is a challenge for operators, especially for different plasma conditions, such as blood viscosity, protein levels, osmotic pressure and so on. ${ }^{\mathbf{1 1 - 1 3}}$ Meanwhile, more errors can be also introduced from using $C_{\mathrm{t}}$ monitoring procedures with another disparate device, such as LLE, PPT. ${ }^{\mathbf{1 4 , 1 5}}$ These methods are unsuitable for $C_{\mathrm{t}}$ monitoring due to unsatisfactory recovery and poor reproducibility obtained from multi-step manipulation procedures, phase change processes and plasma conditions, thus the accuracy of the results needs to be examined as they could mislead the physician in clinical treatment. In addition, two plasma samples collected from each patient are required for $C_{\mathrm{f}}$ and $C_{\mathrm{t}}$ measurement, the collection of which is subject to the compliance of patients. The paired plasma needed is variable due to different collection times and storage conditions, which distorts the analysis result of the PPB and thus it is difficult to faithfully reflect the PPB level in patients in a clinical setting. As a consequence, the above problems assuredly impose restrictions on the application of PPB monitoring in the clinic.

Hollow fiber centrifugal ultrafiltration (HFCF-UF) has been validated as a more accurate means of plasma pre-treatment and has been successfully applied to quantify free CBZ in clinical samples in our previous studies, which has improved on the problems of ED and CF-UF, as described above. ${ }^{16}$ Furthermore, the value of $V_{\mathrm{u}} / V_{\mathrm{s}}$ was correlated to the inside radii of both the glass tube and the hollow fiber. With this smaller and wellcontrolled value, the recovery and precision of the HFCF-UF method were greatly improved and could not be affected by the plasma condition. ${ }^{16,17}$ To make this simple and accurate direct injection technology applicable to routine PPB measurement, by adding an appropriate protein-binding releasing agent into the same plasma following the determination of $C_{\mathrm{f}}$, accurate analysis of $C_{\mathrm{t}}$ in the same system can also be carried out. Therefore, a simplified operation procedure and satisfactory recovery could make the HFCF-UF method feasible and practical to analyze a large number of clinical samples in PPB monitoring.

Carbamazepine (CBZ) is one of most prescribed antiepileptic drugs (AEDs) internationally and is used in the treatment of psychomotor seizures and trigeminal neuralgia. ${ }^{18,19}$ Widespread individual variations of CBZ as well as a narrow therapeutic index range $\left(4-12 \mu \mathrm{g} \mathrm{mL}^{-1}\right)$ have been observed among epilepsy patients, resulting in the occurrence of side and toxic reactions in the clinical response. ${ }^{18}$ Therefore, routine TDM is common and essential for epilepsy patients treated with CBZ in the clinic.

In the present work, we used $\mathrm{CBZ}$ as a representative drug to study the importance of PPB monitoring in routine TDM. To ensure the accuracy of the results and the convenience of the clinical application, a simple pre-treatment method based on hollow fiber centrifugal ultrafiltration (HFCF-UF) for simultaneous $C_{\mathrm{f}}$ and $C_{\mathrm{t}}$ determination in the same plasma samples in a single device was developed and was successfully employed to monitor the PPB of CBZ in clinical plasma samples.

\section{Experimental and methods}

\subsection{Materials and apparatus}

The standard CBZ was purchased from the National Institute for Control of Pharmaceutical and Biological Products (Beijing, China). The hollow fibers $(20 \mu \mathrm{m}$ wall thickness, $10 \mathrm{kDa}$ molecular weight cut-off) were obtained from the Motian Membrane Technology Corporation (Tianjin, China). Methanol (HPLC grade) was from Sigma-Aldrich (Steinheim, Germany). Ultrapure water was prepared by the Milli-Q50 water purification system. All other reagents were of analytical grade. The slim glass tubes $(4.5 \mathrm{~mm}$ outside diameter, $0.5 \mathrm{~mm}$ wall thickness, $7 \mathrm{~cm}$ height) were provided by the Yongda Instrument and Chemical Company (Tianjin, China). Drug-free human plasma was obtained from patients who were not treated with CBZ in the Hebei General Hospital.

\subsection{Chromatographic conditions}

Analysis was performed using an Ultimate 3000 HPLC system consisting of a LPG 3400SD quaternary pump and a VWD-3100 UV detector (Dionex, USA). All of the separations of CBZ were conducted on a Diamonsil $\mathrm{C}_{18}$ column $(150 \mathrm{~mm} \times 4.6 \mathrm{~mm}, 10$ $\mu \mathrm{m}$, Dikma), preceded by a pre-column frit $(4.0 \mathrm{~mm} \times 2.0 \mu \mathrm{m})$ at $30^{\circ} \mathrm{C}$. Chromatography was accomplished using an isocratic elution with methanol and $0.01 \mathrm{~mol} \mathrm{~L}^{-1}$ potassium dihydrogen phosphate buffer solution at $48 / 52(\mathrm{v} / \mathrm{v})$ at a flow rate of $1.0 \mathrm{ml}$ $\mathrm{min}^{-1}$. The detection wavelength was $285 \mathrm{~nm}$ and the injection volume was $20 \mu \mathrm{L}$.

\subsection{Standard solutions and QC sample preparation}

The stock solution ( $535 \mu \mathrm{g} \mathrm{mL}^{-1}$ ) of CBZ was prepared by dissolving the drug in methanol and was used for the preparation of working calibrator samples and working quality control samples (QC). A series of CBZ stock solutions were diluted with pure methanol to achieve final concentrations of 21.4, 10.7, $5.35,2.14,1.07$ and $0.535 \mu \mathrm{g} \mathrm{mL}^{-1}$. The QC samples were 
obtained from a mixture of $450 \mu \mathrm{L}$ of drug-free human plasma and $50 \mu \mathrm{L}$ of three stock solutions to achieve final concentrations of 21.4, 5.35 and $0.535 \mu \mathrm{g} \mathrm{mL}{ }^{-1}$ of total CBZ. The working calibrator samples and QC samples were stored at $4{ }^{\circ} \mathrm{C}$ until use.

\subsection{Sample preparation}

2.4.1 Preparation of free CBZ. A total of $500 \mu \mathrm{L}$ of plasma was subjected to HFCF-UF after the hollow fiber was cut into $15 \mathrm{~cm}$ segments and placed into the tube. After a simple centrifugation at $1.25 \times 10^{3} \mathrm{~g}$ for $15 \mathrm{~min}, 20 \mu \mathrm{L}$ of the ultrafiltrate from inside the hollow fiber was directly injected into the HPLC system. Fig. 1 shows the experimental procedure.

2.4.2 Preparation for obtaining the percentage of the bound drug to protein. Subsequently, $200 \mu \mathrm{L}$ of acetone was added to the same plasma in the same HFCF-UF device. After the mixture was vortexed for $5 \mathrm{~min}$, centrifugation was carried out at $1.25 \times 10^{3} \mathrm{~g}$ for $15 \mathrm{~min}$. The ultrafiltrate $(20 \mu \mathrm{L})$ from the lumen of the hollow fiber was injected into the HPLC system for the analysis of the bound drug. Fig. 2 clearly describes the process.

2.4.3 Calculation for the total CBZ and plasma protein binding. In our previous studies, we have reported that in the HFCF-UF device the ultrafiltrate volume is constant and depends on the inside diameter of the hollow fiber and the glass tube under the conditions of a strong enough centrifugal force and a long enough centrifugation time. ${ }^{16,17}$ Taking into account the inside radii of the glass tube and hollow fiber in our present work, $40 \mu \mathrm{L}$ of ultrafiltrate can be obtained from $500 \mu \mathrm{L}$ of plasma. Based on the volume of the release agent and ultrafiltrate, $C_{\mathrm{t}}$ and PPB were obtained from the following equations:

$$
\begin{gathered}
C_{\mathrm{t}}=\frac{V_{\mathrm{u}} C_{\mathrm{f}}+\left(V_{\mathrm{s}}+V_{\mathrm{r}}-V_{\mathrm{u}}\right) C_{0}}{V_{\mathrm{s}}} \\
\mathrm{PPB}=1-\frac{C_{\mathrm{f}}}{C_{\mathrm{t}}}
\end{gathered}
$$

$C_{\mathrm{f}}$ is the free CBZ concentration in plasma; $C_{\mathrm{t}}$ is the total concentration of $\mathrm{CBZ} ; C_{0}$ is the bound drug concentration of
CBZ in the same plasma and $V_{\mathrm{u}}, V_{\mathrm{s}}$ and $V_{\mathrm{r}}$ represent the volume of the ultrafiltrate in the lumen of the hollow fiber, the plasma samples and the release agent, respectively.

\subsection{Clinical samples}

The study was performed in accordance with the Declaration of Helsinki and the International Conference on the Harmonization Guideline for Good Clinical Practice. The Ethic Review Committee of Hebei Medical University approved all of the procedures (approval no. 2016032). All of the participating patients provided written informed consent. In this study, a total of 20 clinical plasma samples were collected from Hebei General Hospital and were obtained from inpatients (2 male and 4 female; aged 14 months to 79 years) and outpatients (10 male and 4 female; aged 7 months to 63 years) taking CBZ. The subjects were treated with CBZ therapy for more than 1 month, of which the CBZ has reached steady-state plasma concentration. After at least one week of continuous treatment with CBZ, 2-3 $\mathrm{mL}$ of peripheral blood was collected in a centrifuge tube containing heparin just before the morning dose from each patient, and centrifuged at room temperature. Then the obtained plasma samples were stored at $-80{ }^{\circ} \mathrm{C}$ until further analysis.

\subsection{Statistical analysis}

Statistical analysis was performed using SPSS Statistics 21.0. The Wilcoxon signed-rank test was used to compare the mean PPB in epileptic patients with the values from healthy subjects. Linear regression analysis was used to assess the relationship between the total CBZ concentration and the free CBZ concentration, as well as the total CBZ concentrations determined by HFCF-UF and PPT or HFCF-UF and EMIT. A comparison among total CBZ concentration determined by HFCF-UF, PPT and EMIT was done by using two-way classification ANOVA. All statistical tests were two-sided and $p<0.05$ was considered statistically significant.

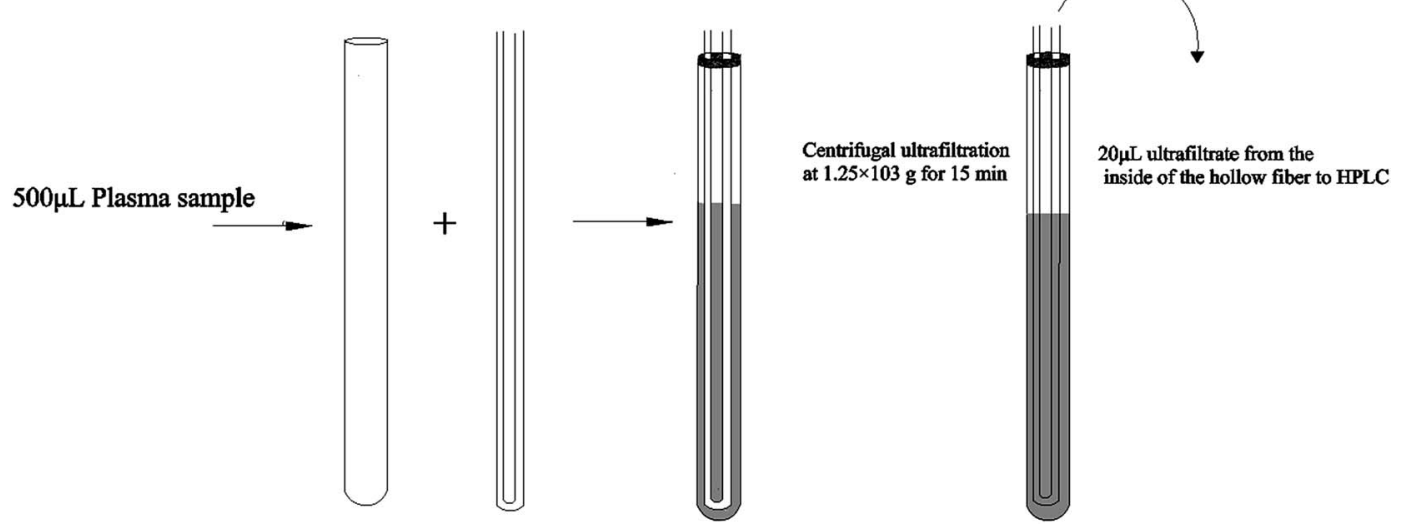

Fig. 1 The experimental procedure for the analysis of free CBZ by HFCF-UF. 


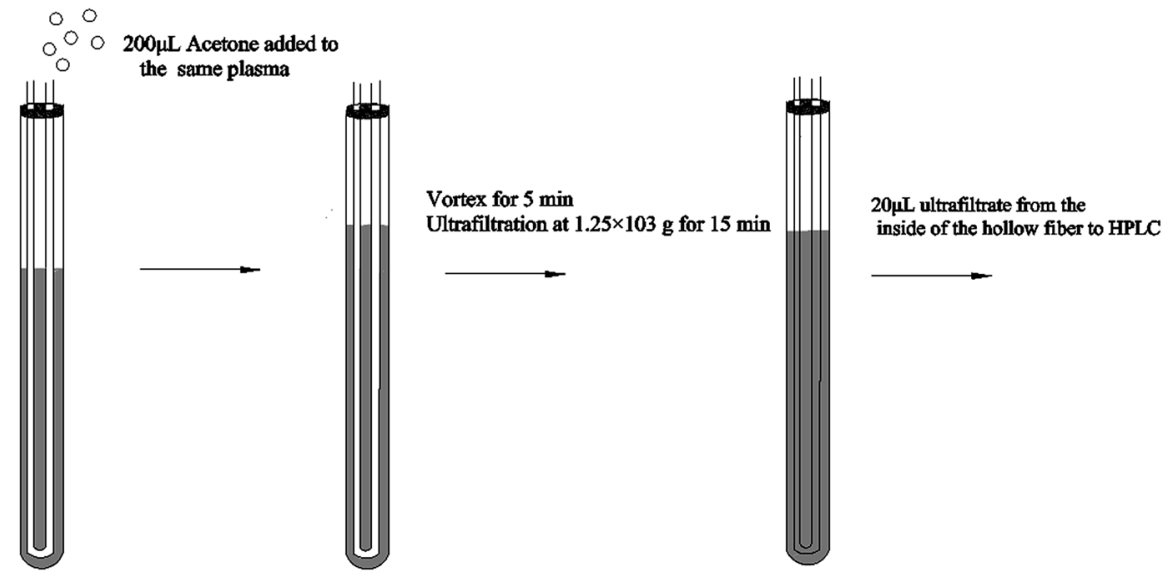

Fig. 2 The preparation procedure of the analysis of the bound concentration by HFCF-UF.

\section{Results and discussions}

\subsection{Method validation}

Method validation was carried out according to the "guidance of the Food and Drug Administration (FDA) for bioanalytical method validation”. ${ }^{20}$ Specificity, linearity, limit of detection, limit of quantification, precision and accuracy, absolute recovery and stability were specifically evaluated.

3.1.1 Specificity. The specificity of this method was evaluated by comparing the chromatogram of blank plasma, human blank plasma spiked with CBZ and a plasma sample from a patient. As shown in Fig. 3, no significant interference was observed at the retention time of CBZ, implying that this method was sufficiently specific. Furthermore, the CBZ capacity factor value of 5.53, the tailing factor value of 0.985 and theoretical plate number of 3865 were calculated according to $\mathrm{ICH}$ guidelines.

3.1.2 Linearity, limit of detection (LOD) and limit of quantification (LOQ). A series of standard solutions with concentrations of $21.4,10.7,5.35,2.14,1.07$ and $0.535 \mu \mathrm{g} \mathrm{mL}^{-1}$ were diluted with pure methanol to generate a calibration curve for $C_{\mathrm{f}}$. Also, a calibration curve for $C_{\mathrm{t}}$ was constructed by plotting the peak area with six different concentrations of CBZ (21.4, 10.7, 5.35, 2.14, 1.07 and $0.535 \mu \mathrm{g} \mathrm{mL}{ }^{-1}$ ) using blank plasma $(450 \mu \mathrm{L})$ spiked with $50 \mu \mathrm{L}$ of the standard working solutions for two replicates of each concentration and then operated as described in the sample preparation section. The linear relationship between the peak area and $C_{\mathrm{f}}$ of CBZ was described by the calibration equation: $A=\left(4.48 \times 10^{4}\right) C-9.52 \times 10^{3}\left(R^{2}=\right.$ 0.9996 , weighted factor $=1 / C^{2}$ ) in the range of $0.535-21.4 \mu \mathrm{g}$ $\mathrm{mL}^{-1}$. The linear relationship between the peak area and $C_{\mathrm{t}}$ in the range of $0.535-21.4 \mu \mathrm{g} \mathrm{mL}^{-1}$ was described by the calibration equation: $A=\left(4.79 \times 10^{4}\right) C-2.26 \times 10^{4}$ with a correlation coefficient of 0.9991 by a weighted factor of $1 / C^{2}$.

The LOD and LOQ were determined by signal-to-noise $(\mathrm{S} / \mathrm{N})$ ratios of 3 and 10, respectively. The LOD and LOQ were 0.214 and $0.535 \mu \mathrm{g} \mathrm{mL}{ }^{-1}$, respectively.

3.1.3 Accuracy, precision and absolute recovery. The accuracy and precision were evaluated by assaying five replicates of
CBZ-spiked plasma $\left(21.4,5.35,0.535 \mu \mathrm{g} \mathrm{mL}{ }^{-1}\right)$. The intra-day precision (coefficient of variation (CV\%)) and accuracy (bias) were evaluated by analyzing five replicates of each concentration on the same day. For the inter-day precision and accuracy, five replicates of each plasma concentration were analyzed on five consecutive days. Both the intra-day and inter-day precision values were less than $3.0 \%$.

The HFCF-UF method was considered a mature approach for the determination of $C_{\mathrm{f}}$ in a previous study because the corresponding absolute recovery was approximately 100\%, therefore, only the recovery of the analysis of $C_{\mathrm{t}}$ by HFCF-UF was examined in this study. ${ }^{16}$ Absolute recovery was assayed by comparing the $C_{\mathrm{t}}$ calculated by eqn (1) with the CBZ standard solution at the same concentrations. Then the PPB was calculated by eqn (2) based on $C_{\mathrm{f}}$ and $C_{\mathrm{t}}$ as described above. Table 1 summarizes the intra-day and inter-day precision as well as the accuracy. The absolute recovery was in the range of $100.2-100.8 \%$ (CV\% < $2.0 \%)$ for $C_{\mathrm{t}}$, showing that this method can satisfy the target drug monitoring of $C_{\mathrm{t}}$ measurements.

3.1.4 Stability. Six aliquots of the $\mathrm{QC}_{\mathrm{S}}$ samples at three concentrations were prepared to investigate the stability of the samples. The stability of the CBZ standard solution and samples after long-term storage and freeze-thaw cycles was investigated. The stability of the standard solution was measured after maintaining the solutions at $4{ }^{\circ} \mathrm{C}$ for a month by comparing the peak areas to those of freshly prepared solutions. The long-term stability was evaluated by comparing the plasma samples with concentrations of $21.4,5.35,0.535 \mu \mathrm{g} \mathrm{mL}{ }^{-1}$, which were kept for 3 months at $-70{ }^{\circ} \mathrm{C}$, with that of the newly prepared samples. In addition, for the analysis of the freezethaw stability, samples were subjected to three cycles of freezing for $24 \mathrm{~h}$ at $-80^{\circ} \mathrm{C}$ and thawing at ambient temperature. All CV\% values for stability were below $4.0 \%$, showing that CBZ remained stable in the whole analysis process.

\subsection{Comparison of $C_{\mathrm{t}}$ from clinical samples by HFCF-UF, PPT and EMIT (Enzyme-Multiplied Immunoassay Technique)}

The $C_{\mathrm{t}}$ of CBZ measured by HFCF-UF, PPT and EMIT were analysed in all of the clinical samples from the 20 patients. As 

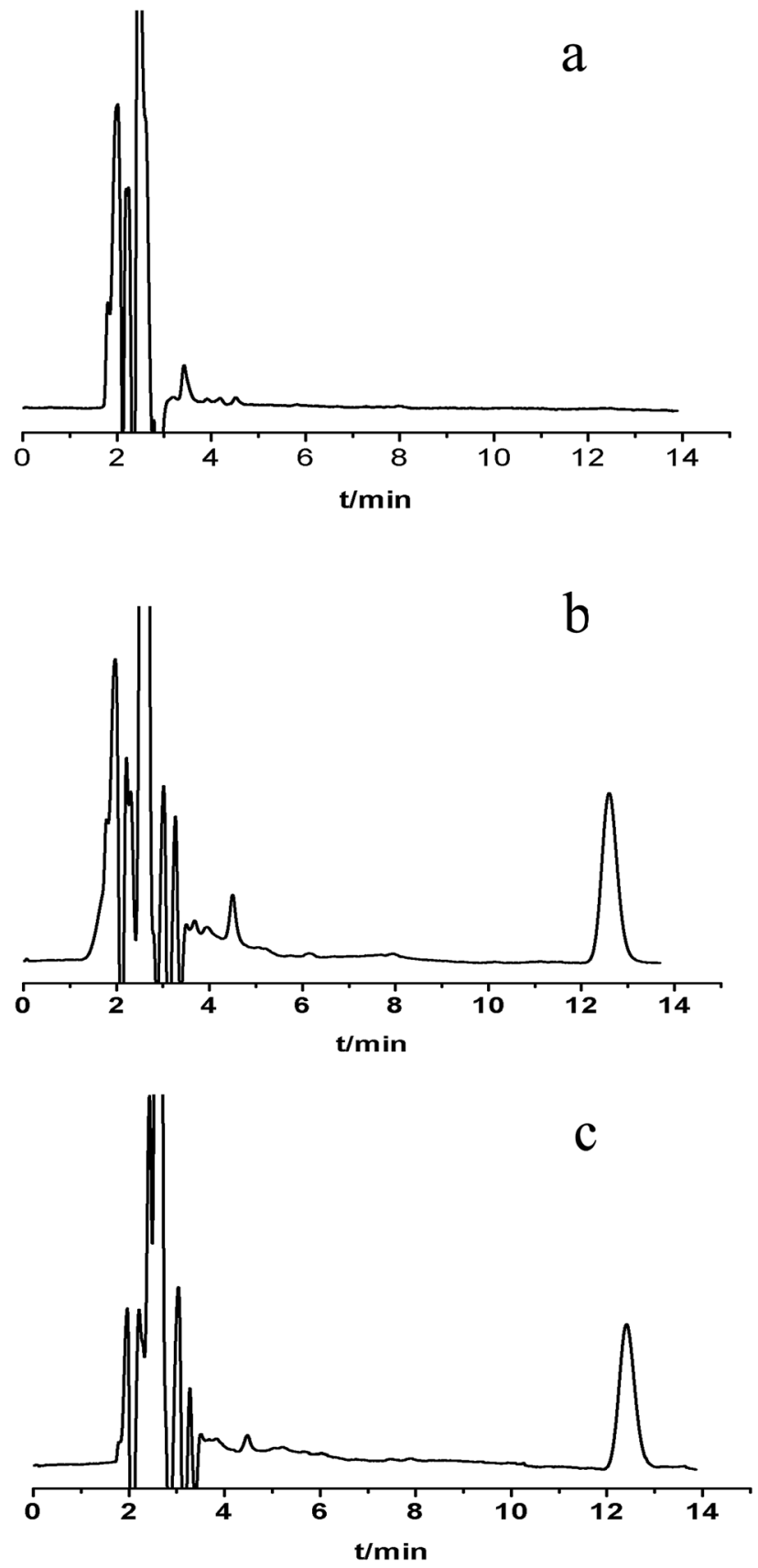

Fig. 3 Typical HPLC chromatograms of the HFCF-UF of drug-free plasma (a), blank plasma spiked with carbamazepine $\left(5.35 \mu \mathrm{g} \mathrm{ml}^{-1}\right)(\mathrm{b})$ and the plasma sample of a patient (c).
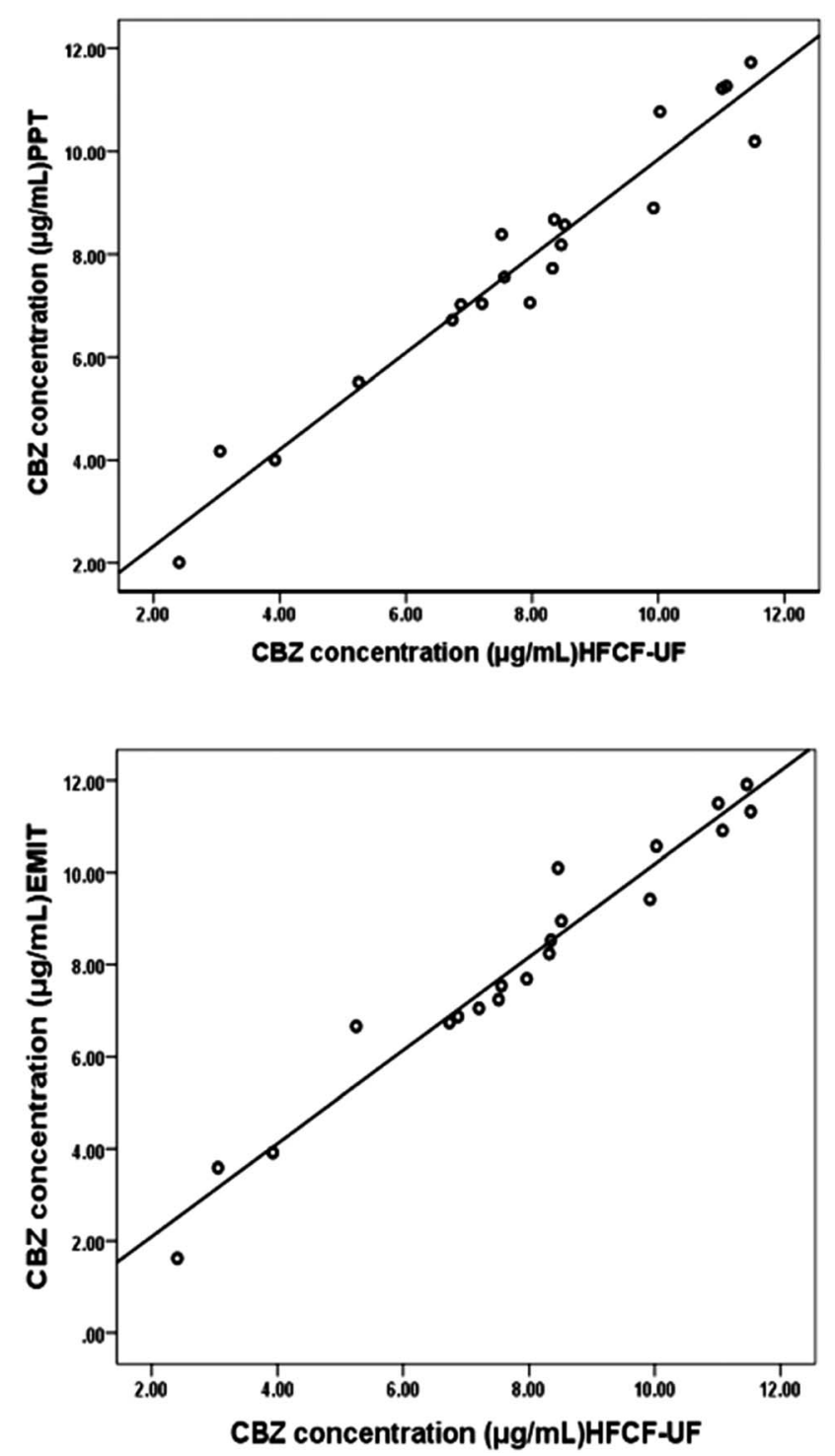

Fig. 4 (a) Correlation between total CBZ concentration determined by HFCF-UF and PPT ( $n=20, y=0.44+0.94 x, r=0.973)$ (b) correlation between total CBZ concentration determined by HFCF-UF and EMIT ( $n=20, y=0.07+1.01 x, r=0.977)$.

Table 1 Intra-day and inter-day accuracy and precision data for total CBZ in plasma

\begin{tabular}{|c|c|c|c|c|c|c|}
\hline $\begin{array}{l}\text { Amount added } \\
\left(\mu \mathrm{g} \mathrm{mL}^{-1}\right)\end{array}$ & $\begin{array}{l}\text { Mean amount } \\
\left(\mu \mathrm{g} \mathrm{mL}^{-1}\right)\end{array}$ & Accuracy $^{a}$ (\% bias) & $\begin{array}{l}\text { Imprecision } \\
(\% \mathrm{CV})\end{array}$ & $\begin{array}{l}\text { Mean amount } \\
\left(\mu \mathrm{g} \mathrm{mL}^{-1}\right)\end{array}$ & Accuracy $^{a}$ (\% bias) & $\begin{array}{l}\text { Imprecision } \\
(\% \mathrm{CV})\end{array}$ \\
\hline 0.535 & 0.530 & -1.0 & 2.2 & 0.533 & -0.4 & 2.9 \\
\hline 5.35 & 5.31 & -0.8 & 1.7 & 5.33 & -0.4 & 1.8 \\
\hline 21.4 & 21.6 & 1.0 & 1.2 & 21.5 & 0.5 & 1.5 \\
\hline
\end{tabular}

${ }^{a}$ Accuracy $=[($ mean amount - added amount $) /$ added amount $] \times 100$. 
shown in Fig. 4, comparison of the proposed HFCF-UF method with either PPT or EMIT showed good agreement which confirmed the accuracy of the HFCF-UF method. In addition, there were no significant differences among the results determined by the three methods $(p>0.05)$. Thus, the HFCF-UF method for measuring $C_{\mathrm{t}}$ could be comparable with the widely accepted methods - PPT and EMIT - but the proposed HFCF-UF method was the simplest and most accurate among the three methods.

For the PPT method, a relative ratio of $300 \mu \mathrm{L}$ of methanol to $100 \mu \mathrm{L}$ of plasma sample was required to precipitate proteins, which led to the effects of dilution and low detection sensitivity. Furthermore, the target drug was prone to be adsorbed and embedded by the precipitated protein, which resulted in a loss of the drug and a reduction in recovery. Moreover, there was variation among recoveries in the phase transition process due to the differences in operation processes (vortex time, centrifugal speed and so on) and the condition of plasma in epileptics (blood viscosity, protein level and so on). Thus, the measured $C_{\mathrm{t}}$ often deviated from the true values, which misled the physician who was optimizing epileptic patients' drug regime, and further affected clinical treatment. Therefore, a simple and accurate assay for $C_{\mathrm{t}}$ analysis is desired to make it feasible and practical to analyze a large number of clinical samples. In the present work, the validated HFCF-UF method only required $200 \mu \mathrm{L}$ of acetone as the protein-binding releasing agent into $500 \mu \mathrm{L}$ of the plasma. The volume of organic solvent in HFCF-UF was significantly lower than that in PPT, making the sensitivity of HFCF-UF higher than that of PPT. Also, acetone could almost completely release CBZ from the protein into the plasma without a phase transition process and all small drug molecules could pass though membrane freely without any concentration polarization phenomenon by ultrafiltration, as the direction of centrifugal force is completely parallel to the membrane. As a consequence, higher recovery and reproducibility were obtained by this simple method, meeting the need of monitoring the total CBZ in clinical plasma samples in routine TDM.

As for EMIT, the data for $C_{\mathrm{t}}$ in this work was determined by the experienced clinical pharmacist from Hebei General Hospital. The operation process was carried out according to the corresponding Emit CBZ assay instruction on a Syva Autolab System. Meanwhile, determination of CBZ in plasma or serum has been developed by Jens Martens (1993) and B. Rambeck et al. (1994)..$^{21,22}$ This method has been a traditional and simple one for in vitro diagnostic use in the quantitative analysis of CBZ in a hospital. However, the process of EMIT suffered from many influencing factors (temperature, reaction time and so on). Julia Grandke et al. reported that incubation temperature, as a factor in immunoassays, greatly influenced the assay sensitivity of CBZ, especially in the case of concomitant medication. ${ }^{23}$ Therefore, rigorous operation as well as controlling the impact is key to achieve the high performance of the immunoassay in routine CBZ monitoring. In addition, the overestimation of the target drug concentration using EMIT has been presented in the literature and is due to not only unspecific cross-reactivity but also plasma condition. ${ }^{24}$ In contrast, the pre-treatment in the HFCF-UF method was simple as it just involves the centrifugal operation, which is not susceptible to environmental factors, so this method has excellent reproducibility, is easier to perform and consumes less time, especially for a large number of biological samples.

In addition, this method had a conspicuous predominance on the simultaneous analysis of $C_{\mathrm{f}}$ and $C_{\mathrm{t}}$ in one plasma sample without other pretreatment for $C_{\mathrm{f}}$. The errors resulting from multi-step manipulation procedures, as well as the use of two plasma samples, were largely reduced, which could sufficiently meet the needs of TDM in patient management.

\subsection{Data analysis of clinical samples}

The proposed HFCF-UF method was successfully applied to determine the PPB in 20 patients undergoing treatment with CBZ. The ranges of $C_{\mathrm{t}}$ and $C_{\mathrm{f}}$ were $2.41-11.5 \mu \mathrm{g} \mathrm{mL} \mathrm{m}^{-1}$ and 1.07$10.3 \mu \mathrm{g} \mathrm{mL} \mathrm{m}^{-1}$. The PPB data of each patient is shown in Fig. 5 and the mean (range) of PPB was $46.3 \%$ (10.1-68.9\%). Out of all of the observations, 17 of the $C_{\mathrm{t}}$ measurements were within the therapeutic range of $4-12 \mu \mathrm{g} \mathrm{mL}^{-1}$. There was a statistical difference in the data of PPB among the patients and in addition, between the patients and healthy people $(p<0.05)$. The results indicated that in clinical practice, using the PPB (approximately 76\%) from healthy people to calculate $C_{\mathrm{f}}$ on the basis of determined $C_{\mathrm{t}}$ to guide dose adjustments is irrational.

As shown in Fig. 6, there was a weak correlation between $C_{\mathrm{t}}$ and $C_{\mathrm{f}}$. The increase in $C_{\mathrm{f}}$ was not associated with an increase in $C_{\mathrm{t}}$. One patient's $C_{\mathrm{t}}$ of CBZ was in the therapeutic range at 8.32 $\mu \mathrm{g} \mathrm{mL}{ }^{-1}$, while their $C_{\mathrm{f}}$ was high at $7.48 \mu \mathrm{g} \mathrm{mL}{ }^{-1}$. Another patient's $C_{\mathrm{t}}$ of CBZ was lower than the therapeutic range at 3.92 $\mu \mathrm{g} \mathrm{mL}{ }^{-1}$, whereas their free fraction was high at $64 \%$. Thus, the $C_{\mathrm{f}}$ of CBZ cannot be estimated from $C_{\mathrm{t}}$. Due to the difference in sampling time, the $C_{\mathrm{f}}$ measured at one time was not capable of predicting the CBZ concentration at another time, and also the extent of increasing or decreasing the drug dose could not be deduced from the single $C_{\mathrm{f}}$ measurement. So it is essential to choose a correct dose metric in TDM for individual drug delivery.

A drug binding to plasma protein (PPB) is a reversible process, which may be in a steady-state equilibrium with plasma under stable physiological conditions. In addition, an

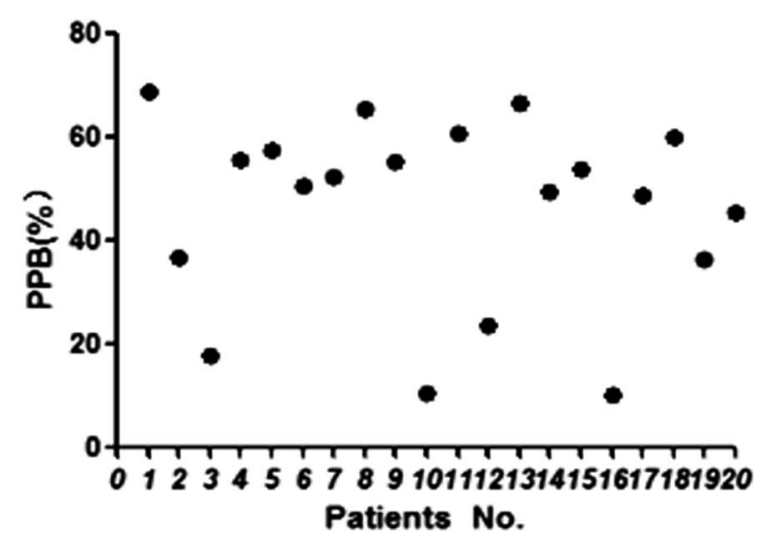

Fig. 5 The PPB data of 20 patients. 


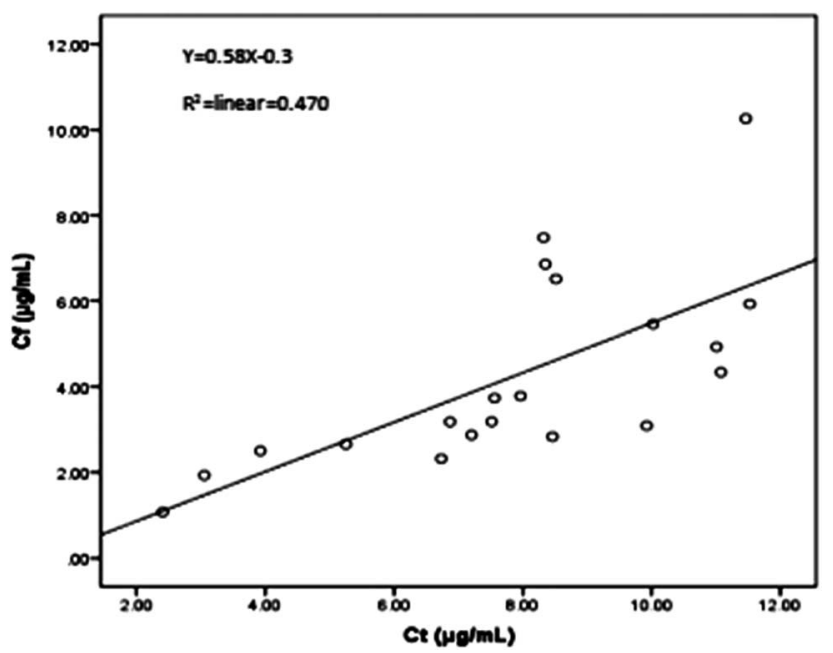

Fig. 6 Correlation between free and total CBZ concentration in 20 clinical plasma samples.

individual variation of PPB directly results in a change of $C_{\mathrm{f}}$, which further influences the therapeutic effects of CBZ. For this reason, the optimisation of dosing regimens to avoid side effects could be based on PPB in TDM. In the present work, we have successfully applied PPB monitoring into TDM.

\subsection{Assessment of non-specific binding (NSB) in ultrafiltration}

The major disadvantage of the UF method is the non-specific binding of drugs to the filter membrane, leading to the ultrafiltrate concentration deviating from the true drug concentration. Therefore, an evaluation of NSB was needed before obtaining PPB measurements by HFCF-UF devices. Five replicates of CBZ standard solution were prepared at three concentrations $\left(21.4,5.35\right.$ and $0.535 \mu \mathrm{g} \mathrm{mL}^{-1}$ ), then $500 \mu \mathrm{L}$ was placed into the HFCF-UF devices and centrifuged at $1.25 \times 10^{3} \mathrm{~g}$ for 15 min. Finally, intra- and extra- solutions of three types of hollow fiber (polyvinylidene fluoride, polysulfone and polyamide) were injected into the HPLC system. The NSB in ultrafiltration was assessed by comparing the obtained concentrations of the extra-solution, as well as the intrasolutions, of the hollow fiber to the corresponding concentration of the standard solutions. The results were all around $100 \%$ $(\mathrm{CV} \%<3.0 \%)$, indicating that there was no significant NSB in this study. Considering the flexibility and purchasing convenience of the three kinds of hollow fibers, we chose the polyvinylidene fluoride hollow fiber to be used for further study.

\subsection{Selection of the protein-binding releasing agent}

The key to determining PPB using the HFCF-UF method is to select a stable protein-binding releasing agent to completely release CBZ from the drug-bound fraction and dissolve the small drug molecules across the membranes freely. Thus, both $C_{\mathrm{f}}$ and $C_{\mathrm{t}}$ could be obtained from the same plasma. As the target drug (CBZ) is a kind of liposoluble protein-binding drug, several organic solvents, such as methanol, acetonitrile, ethanol and acetone were discussed in the present study. The results are

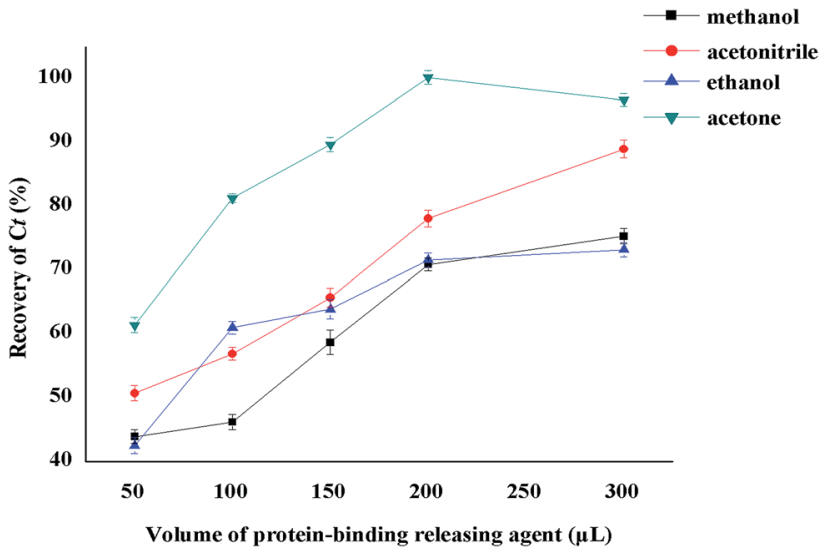

Fig. 7 The effect of the type and volume of protein-binding releasing agents on the recovery of $C_{t}$.

shown in Fig. 7. The recovery of $C_{\mathrm{t}}$ made it clear that acetone was the most appropriate protein-binding releasing agent out of the above solutions, with the highest recovery of CBZ. Thus, acetone was used in the following experiments.

The effect of different protein-binding releasing agent volumes on the extent of CBZ released from the drug-bound fraction was also investigated (Fig. 7). The volume of organic solvents used was also associated with the sensitivity. The best recovery of $C_{\mathrm{t}}$ was obtained when the volume of acetone reached $200 \mu \mathrm{L}$. Additionally, the volume of acetone increased with decreasing sensitivity. In contrast, unsatisfactory recovery was a result of decreasing the releasing agent volume. So, the best volume of acetone was $200 \mu \mathrm{L}$ and this volume was used in the experiments.

\section{Conclusion}

In the present study, the significance of a new parameter - PPB in TDM was presented and successfully applied to epileptics taking CBZ. Compared $C_{\mathrm{f}}$ or $C_{\mathrm{t}}$, using PPB to guide the individual dose adjustment was more rational. Meanwhile, we developed a simple and accurate method based on HFCF-UF coupled with HPLC for monitoring the PPB of CBZ and found that $C_{\mathrm{t}}$ and $C_{\mathrm{f}}$ could both be obtained from one clinical plasma sample in a single HFCF-UF device. This method had a conspicuous predominance on the analysis of $C_{\mathrm{t}}$ and $C_{\mathrm{f}}$ with excellent accuracy and precision. In addition, the developed method achieved successful application in the TDM of 20 Chinese epilepsy patients undergoing CBZ therapy. Therefore, it would be a simple and reliable alternative to PPB analysis of biological samples in routine TDM.

\section{Acknowledgements}

The authors gratefully acknowledge financial support from the Key Research Project of Hebei Province in China 2017 (project no. 17277740D). 


\section{References}

1 S. M. Illamola, D. Hirt, J. M. Treluyer, S. Urien and S. Benaboud, Challenges regarding analysis of unbound fraction of highly bound protein antiretroviral drugs in several biologica matrices: lack of harmonisation and guidelines, Drug Discovery Today, 2014, 1-9.

2 A. Dasgupta, Usefulness of monitoring free (unbound) concentrations of therapeutic drugs in patient management, Clin. Chim. Acta, 2007, 377, 1-13.

3 K. A. Mortier and W. E. Lambert, Determination of unbound docetaxel and paclitaxel in plasma by ultrafiltration and liquid chromatography-tandem mass spectrometry, $J$. Chromatogr. A, 2006, 1108, 195-201.

4 Y. Dou, J.-W. Guo, Y. Chen, S.-L. Han, X.-Q. Xu, Q. Shi, Y. Jia, Y. Liu, Y.-C. Deng, R.-B. Wang, X.-H. Li and J.-X. Zhang, Sustained delivery by a cyclodextrin material-based nanocarrier Potentiates antiatherosclerotic activity of rapamycin via selectively inhibiting mTORC1 in Mice, $J$. Controlled Release, 2016, 235, 48-62.

5 J. Goole, B. V. Gansbeke, G. Pilcer, P. Deleuze, D. Blocklet, S. Goldman, M. Pandolfo, F. Vanderbist and K. Amighi, Pharmacoscintigraphic and pharmacokinetic evaluation on Healthy human volunteers of sustained-release floating minitablets containing levodopa and carbidopa, Int. J. Pharm., 2008, 364, 54-63.

6 J. J. Marty, C. J. Kilpatrick and R. F. W. Moulds, Intra-dose variation in plasma protein binding of sodium valproate in epileptic patients, Br. J. Clin. Pharmacol., 1982, 14, 399-404.

7 R. T. Heine, E. M. V. Maarseveen, M. L. Monique, K. P. J. Braun, S. M. Koudijs, M. J. Berg and M. M. Malingre, The Quantitative Effect of Serum Albumin, Serum Urea, and Valproic Acid on Unbound Phenytoin Concentrations in Children, J. Child Neurology, 2013, 6, 803-810.

8 F.-L. Zhang, J.-P. Xue, J.-W. Shao and L. Jia, Compilation of 222 drugs' plasma protein binding data and guidance for study designs, Drug Discovery Today, 2012, 17, 475-485.

9 P. Ascenzi, G. Fanali, M. Fasano, V. Pallottini and V. Trezza, Clinical relevance of drug binding to plasma proteins, J. Mol. Struct., 2014, 1077, 4-13.

10 R. H. Levy and M. S. Yerby, Effects of Pregnancy on Antiepileptic Drug Utilization, Epilepsia, 1985, 26, 52-57.

11 W.-C. Dong, J.-F. Zhang, Z.-L. Hou, X.-H. Jiang, C.-F. Zhang, F.-H. Zhang and Y. Jiang, The influence of volume ratio of ultrafiltrate of sample on the analysis of non-protein binding drugs in human plasma, Analyst, 2013, 138, 7368-7375.

12 L. Zhang, Z.-Q. Zhang, W.-C. Dong, S.-J. Jing, J.-F. Zhang and Y. Jiang, Accuracy assessment on the analysis of unbound drug in plasma by comparing traditional centrifugal ultrafiltration with hollow fiber centrifugal ultrafiltration and application in pharmacokinetic study, J. Chromatogr. A, 2013, 1318, 265-269.

13 J.-M. Li, Y. Jiang, T. Sun and L.-J. Kang, Hollow-fiber ultrafiltration then centrifugation for LC analysis of watersoluble sucrose in a water-soluble high-molecular-mass gel matrix, J. Chromatogr., 2009, 70, 1023-1030.
14 H. A. Mowafy, F. K. Alanazi and G. M. Maghraby, Development and validation of an HPLC-UV method for the quantification of carbamazepine in rabbit plasma, Saudi Pharm. J., 2012, 20, 29-34.

15 M. Behbahani, F. Najafi, S. Bagheri, M. K. Bojdi, M. Salarian and A. Bagheri, Application of surfactant assisted dispersive liquid-liquid microextraction as an efficient sample treatment technique for preconcentration and trace detection of zonisamide and carbamazepine in urine and plasma samples, J. Chromatogr. A, 2013, 1308, 25-31.

16 W.-C. Dong, Z.-Q. Zhang, X.-H. Jiang, Y.-G. Sun and Y. Jiang, Effect of volumeratio of ultrafiltrate to sample solution on the analysis of free drug and measurement of free carbamazepine in clinical drug monitoring, Eur. J. Pharm. Sci., 2013, 48, 332-338.

17 J.-F. Zhang, X.-L. Yang, Z.-Q. Zhang, W.-C. Dong and Y. Jiang, Accuracy of the analysis of free vancomycin concentration by ultrafiltration in various disease states, RSC Adv., 2014, 4, 40214-40222.

18 E. Shokry, F. Villanelli, S. Malvagia, A. Rosati, G. Forni, S. Funghini, D. Ombrone, M. D. Bona, R. Guerrini and G. L. Marca, Therapeutic drug monitoring of carbamazepine and its metabolite in children from dried blood spots using liquid chromatography and tandem mass spectrometry, J. Pharm. Biomed. Anal., 2015, 109, 164-170.

19 A. J. Ajura, S. H. Lau and S. Nur-Suffia, An observational study of trigeminal neuralgia patients taking carbamazepine during the fasting month of ramadan, Med. J. Malays., 2016, 6, 305-307.

20 U.S. Department of Health and Human Services, Food and Drug Administration, Center for Drug Evaluation and Research (CDER), Center for Veterinary Medicine (CVM), Guidance for Industry, Bioanalytical Method Validation, 2001.

21 B. Rambeck, T. W. May, M. U. Jurgens, V. Blankenhorn, U. Jurges, E. Korn-Merker and A. Salke-Kellermann, Comparison of phenytoin and carbamazepine serum concentrations measured by high-performance liquid chromatography, the standard TDx assay, the enzyme multiplied immunoassay technique, and a new patientside immunoassay cartridge system, Ther. Drug Monit., 1994, 16, 608-612.

$22 \mathrm{~J}$. Martens and P. Banditt, Validation of the analysis of carbamazepine and its 10,11-epoxide metabolite by highperformance liquid chromatography from plasma: comparison with gas chromatography and the enzymemultiplied immunoassay technique, J. Chromatogr., 1993, 620, 169-173.

23 J. Grandke, U. Resch-Genger, W. Bremser, L. A. Garbeb and R. J. Schneider, Quality assurance in immunoassay performance-temperature effects, Anal. Methods, 2012, 4, 901-905.

24 I. R. Dubbelboer, A. Pohanka, R. Said, S. Rosenborg and O. Beck, Quantification of Tacrolimus and Three Demethylated Metabolites in Human Whole Blood Using LC-ESI-MS/MS, Ther. Drug. Monit, 2012, 34, 134-142. 